\title{
STOCHASTIC ANALYSIS OF FRACTIONAL OSCILLATORS BY EQUIVALENT SYSTEM DEFINITION
}

\author{
Giorgio Barone ${ }^{1}$, Alessandro Palmeri ${ }^{1}$, and Mariateresa Lombardo ${ }^{1}$ \\ ${ }^{1}$ School of Civil and Building Engineering, Loughborough University \\ Loughborough, LE11 3TU, UK \\ \{G.Barone, A.Palmeri, M.Lombardo\}@lboro.ac.uk
}

Keywords: Fractional calculus, Fractional oscillator, White Noise, Stochastic Analysis.

\begin{abstract}
Fractional oscillators have been recently proposed as damping devices under the configuration of Fractional Tuned Mass Dampers (FTMD), realized by connecting an oscillating mass to the primary structure through a viscoelastic link with inherent fractional constitutive law. The characteristic tuning frequency for the FTMD has been identified with the Damped Fractional Frequency (DFF), defined as the frequency at which the squared absolute value of the transfer function of the device attains its relative maximum. The definition of the DFF constitutes an interesting step towards the analysis of fractional oscillators in the frequency domain. In this paper, a simplified frequency domain approach is presented for the design of fractional oscillators subjected to stationary white noise. The analysis of the fractional oscillator is performed by using an equivalent single degree of freedom system with linear viscous damping. The aim is to obtain a clear understanding of the physical dynamic effects of the variations in the fractional oscillator parameters, in terms of damping and natural frequencies. Moreover, the use of an equivalent system allows for the straightforward applications of stochastic analysis to determine an approximate closed-form expression of the response variance.
\end{abstract}




\section{INTRODUCTION}

The theoretical basis for accurately modeling viscoelastic materials using concepts of fractional calculus came into view starting from the beginning of the $20^{\text {th }}$ century [1]. Since then, several researchers have used fractional integrals and derivatives to model the constitutive law of viscoelastic materials [2-5]. Due to the increased use of fractional models in physics and engineering applications, several approaches have been proposed to numerically represent fractional derivatives and integrals and to determine the motion of oscillatory systems with inherent fractional terms [6-9].

Recently, the use of fractional oscillators configured as Fractional Tuned Mass Dampers (FTMD) has been proposed in [10-12]. In this context, a novel approach to the analysis of fractional systems has been proposed, leading to the definition of the Damped Fractional Frequency (DFF). This different perspective on fractional dynamic systems, however, still requires further in-depth analysis. In particular, in this paper, the same fractional oscillators are analyzed in the frequency domain by means of equivalent systems with linear viscous damping. The aim is to establish direct relationships between the parameters of the two systems (fractional and equivalent) in order to extend, to the fractional case, the use of the mathematical tools well-established for the case of linear viscous damping. In the following, the characteristic parameters of the equivalent system are derived analytically from the parameters of the fractional one. Then, a numerical procedure is proposed for the solution of the inverse design problem, i.e. the calculation of the fractional system parameters for an assigned equivalent system. Finally, the proposed tools are used to determine an approximate closed-form expression for the variance of the response of a fractional oscillator excited by a zero-mean Gaussian white noise.

\section{THE FRACTIONAL OSCILLATOR IN THE FREQUENCY DOMAIN}

In this paper, the term fractional oscillator refers to a single degree-of-freedom system composed by a mass $m$ connected to a fixed support by means of a viscoelastic link with fractional properties [10], as shown in Fig.1. In the hypothesis of small displacements of the mass and, therefore, linear behavior of the system, the dynamic equilibrium of the system can be expressed by the following equation of motion:

$$
m \ddot{x}(t)+C_{\beta}\left({ }^{C} D_{0^{+}}^{\beta} x\right)(t)=F(t)
$$

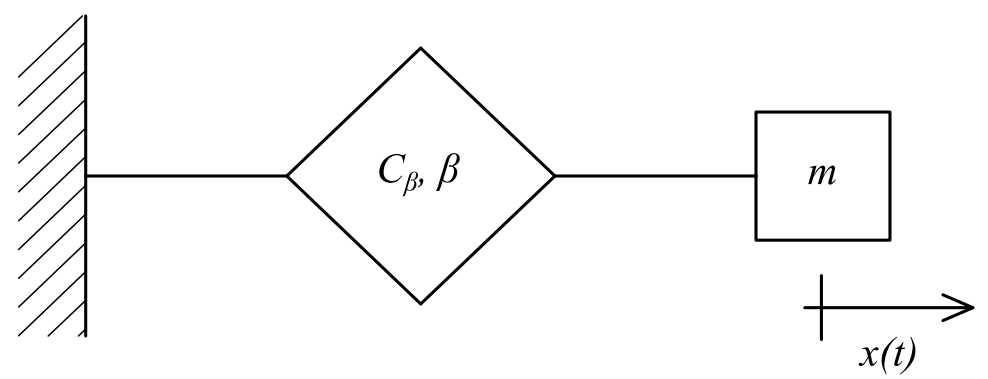

Figure 1: Fractional oscillator.

In eq.(1), $C_{\beta}$ is the fractional damping coefficient. The elastic and viscous terms are reduced to a single fractional term through the Caputo's fractional derivative of the displacement of the mass:

$$
\left({ }^{C} D_{0^{+}}^{\beta} x\right)(t)=\frac{1}{\Gamma(1-\beta)} \int_{0}^{t}(t-\bar{t})^{-\beta} \dot{x}(\bar{t}) d \bar{t}
$$

where $\Gamma($.$) is Euler's Gamma function. By dividing eq.(1) for the mass m$ of the system, the equation of motion can be rewritten in canonical form as: 


$$
\ddot{x}(t)+\eta\left({ }^{C} D_{0^{+}}^{\beta} x\right)(t)=f(t)
$$

where $\eta=C_{\beta} / m$ and $f(t)=F(t) / m$. The system in eq.(3) is characterized by the two parameters $\eta$ and $\beta$.

Due to the peculiar form of the equation of motion, the classic definition of natural and damped frequencies cannot be adopted. A novel formulation in the frequency domain has recently been proposed in [10-12], based on the analysis of the transfer function of the fractional oscillator:

$$
H_{f}(\omega)=\frac{1}{-\omega^{2}+\eta(i \omega)^{\beta}}
$$

From a critical examination of the square modulus of $H_{f}(\omega)$, depicted in Fig.2 in the positive frequency domain and for an arbitrary value of the coefficient $\eta$ and various $\beta$, two behaviors of the fractional system can be distinguished with respect to a critical value $\beta_{c}$. In the first case $\left(\beta>\beta_{c}\right)$, the transfer function decreases monotonically, similarly to overdamped systems with linear viscous damping. However, if $\beta<\beta_{c}$, a relative maximum is observed in the transfer function, i.e. the system exhibits a prevalent elastic resonant behavior. The critical value $\beta=\beta_{c}$, corresponding to the case of transfer function with horizontal tangent flexure point, has been identified in [10] as $\beta_{c} \cong 0.44$.

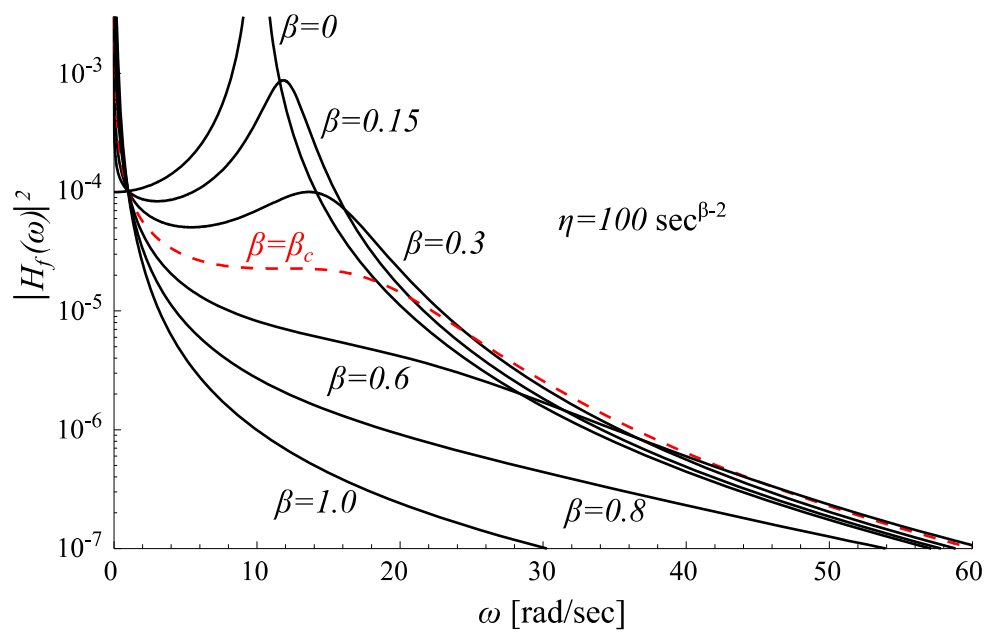

Figure 2: Fractional oscillator transfer function.

For the case of dominant elastic dynamic behavior, the DFF of the system has been defined as the frequency at which the squared absolute value of the transfer function attains its (relative) maximum:

$$
\omega_{f}=\left(\frac{\eta}{\theta(\beta)}\right)^{\frac{1}{2-\beta}}
$$

where the function $\theta(\beta)$ is expressed as:

$$
\theta(\beta)=\frac{(2+\beta) \cos \left(\frac{\pi \beta}{2}\right)-\sqrt{\left((2+\beta) \cos \left(\frac{\pi \beta}{2}\right)\right)^{2}-8 \beta}}{2 \beta}
$$


The DFF has been determined by equating to zero the first derivative of the squared absolute value of the transfer function in eq.(4), and the detailed algebra to achieve eqs.(5) and (6) can be found in [10].

\section{EQUIVALENT SYSTEM WITH LINEAR VISCOUS DAMPING}

In this section, an equivalent system is defined for the fractional system considered in the previous section. In the following, the term equivalent is used to identify a system with linear viscous damping and whose transfer function exhibits its peak at the same frequency and with the same magnitude of the fractional system. Indicating with $\omega_{0}$ the natural frequency of the equivalent system and with $\zeta$ its damping coefficient, the transfer function $H_{e}(\omega)$ of the equivalent system is defined as:

$$
H_{e}(\omega)=\frac{1}{\omega_{0}^{2}-\omega^{2}+2 i \zeta \omega_{0} \omega}
$$

Of course, different definitions of equivalent systems could be used, considering different criteria. Herein, the final aim is to achieve a better understanding of the effects of the variations of the parameters $\eta$ and $\beta$ of the fractional oscillator in terms of the equivalent parameters $\omega_{0}$ and $\zeta$. Also, as shown in the next sections, such definition of equivalent system leads to an approximate closed-form equation for the evaluation of the variance of the response of the fractional system.

To determine analytic relations between the parameters $(\eta, \beta)$ of the fractional system and the parameters $\left(\omega_{0}, \zeta\right)$ of the equivalent system, two algebraic equations have to be considered. As already mentioned, the first condition required for the equivalent system is that the peaks in the transfer functions of the two systems occur at the same frequency. For the fractional oscillator, this frequency has been identified as the DFF $\omega_{f}$, while for the equivalent system it is well-known that the peak of the transfer function occurs at the frequency $\omega_{0} \sqrt{1-2 \zeta^{2}}$ [13]. Therefore, taking into account eq.(5), the first relation between the parameters of the two systems can be written as:

$$
\omega_{0} \sqrt{1-2 \zeta^{2}}=\left(\frac{\eta}{\theta(\beta)}\right)^{\frac{1}{2-\beta}}
$$

The second condition given for the equivalent system is that the amplitudes of the transfer functions of the two systems at the previously indicated frequencies $\left(\omega_{f}\right.$ and $\left.\omega_{0} \sqrt{1-2 \zeta^{2}}\right)$ are the same, i.e.:

$$
\left|H_{e}\left(\omega_{0} \sqrt{1-2 \zeta^{2}}\right)\right|^{2}=\left|H_{f}\left(\omega_{f}\right)\right|^{2}
$$

By solving the system composed by the two eqs.(8) and (9) in the unknown $\omega_{0}$ and $\zeta^{2}$, four couple of solutions $\left(\omega_{0}, \zeta^{2}\right)$ are obtained. Each solution describes the parameters $\left(\omega_{0}, \zeta^{2}\right)$ as function of the parameters of the fractional oscillator $(\eta, \beta)$. However, among the four possible solutions, only one returns both $\omega_{0} \in \mathbb{R}$ and $\zeta^{2} \geq 0$. The latter leads to the following two relations:

$$
\begin{aligned}
& \omega_{0}=\left(\frac{\eta}{\theta(\beta)}\right)^{\frac{1}{2-\beta}} \psi(\beta) \\
& \zeta^{2}=\frac{1}{2}\left(1-\psi(\beta)^{-2}\right)
\end{aligned}
$$

where the function $\psi(\beta)$ is defined as: 


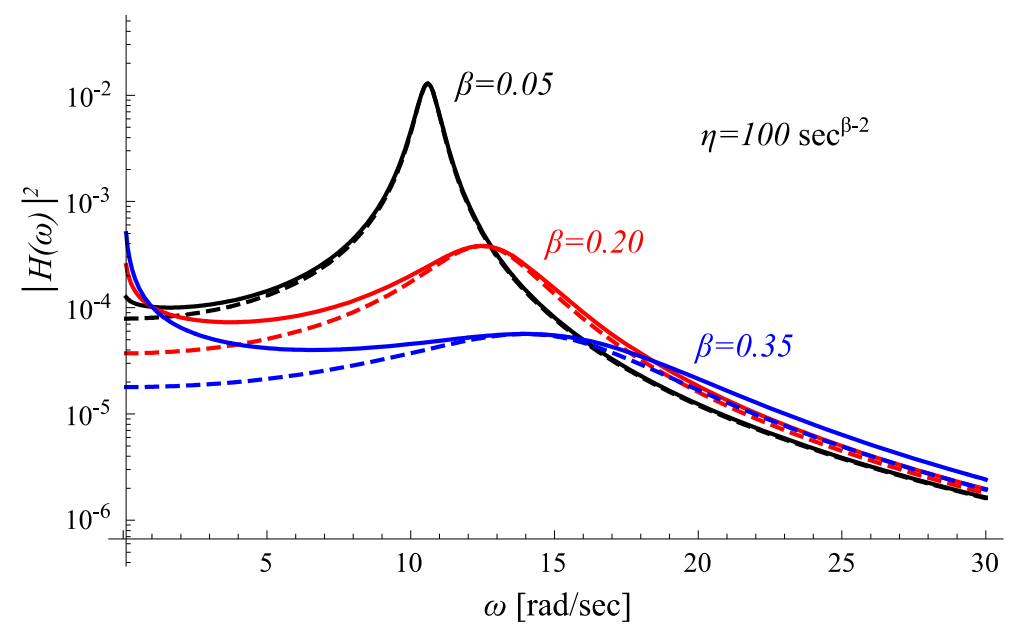

Figure 3: Transfer functions of fractional (continuous) and equivalent systems (dashed) reported in Table 1.

$$
\psi(\beta)=\left(\theta(\beta)^{2}-2 \theta(\beta) \cos \left(\frac{\pi \beta}{2}\right)+2\right)^{\frac{1}{4}}
$$

and $\theta(\beta)$ is given in eq.(6). Hence, the equivalent damping coefficient $\zeta$ depends only on the parameter $\beta$, while the equivalent natural frequency $\omega_{0}$ depends on both the fractional parameters $(\eta, \beta)$. Fig. 3 shows the comparison among the transfer functions of three fractional systems and their equivalent systems obtained by using eqs.(10). The three fractional systems are defined considering the same value for the parameter $\eta$ and increasing values of $\beta$ (with $\beta<\beta_{c}$ ). Values of these parameters and the natural frequencies and damping coefficients for the corresponding equivalent systems are reported in Table 1 . As expected, the differences between the transfer function of each fractional system and its equivalent one increase when larger values of $\beta$ are considered, since the behavior of the fractional system shifts towards the pure viscous behavior when $\beta \rightarrow \beta_{c}$.

\begin{tabular}{|c|c|c|c|}
\hline$\eta\left[\sec ^{\beta-2}\right.$ & $\beta$ & $\omega[\mathrm{rad} / \mathrm{sec}]$ & $\zeta$ \\
\hline 100 & 0.05 & 10.607 & 0.039 \\
\hline 100 & 0.20 & 12.802 & 0.158 \\
\hline 100 & 0.35 & 15.375 & 0.294 \\
\hline
\end{tabular}

Table 1: Fractional systems parameters and corresponding equivalent systems paramaters.

\section{DESIGN OF FRACTIONAL OSCILLATOR WITH ASSIGNED EQUIVALENT SYSTEM}

The determination of the equivalent system for a given fractional oscillator is quite straightforward by using eqs.(10) and (11). However, the inverse problem, that is to determine the fractional system parameters for a given equivalent system, is not as simple as the direct one. The non-trivial inverse problem can be described, in other words, as the design of the fractional oscillator parameters $(\eta, \beta)$, so that its transfer function has maximum at an assigned frequency and with assigned amplitude. In principle, the exact solution of this problem would be obtained by using the inverse of eqs.(10). However, due to the nonlinearity of the function $\theta(\beta)$, expressed in eq.(6), it has not be possible, at this stage, to determine the closed-form expression of $(\eta, \beta)$ for assigned parameters $\left(\omega_{0}, \zeta\right)$. In the following, a numerical iterative procedure is proposed to obtain the value of $\beta$ at first. Then, $\eta$ can be easily obtained by means of the inverse of eq.(8). 
At the $i$-th step of the iterative procedure a tentative value $\tilde{\beta}_{i}$ is obtained by taking advantage of eqs.(10) as:

$$
\tilde{\beta}_{i}=\frac{2}{\pi} \arccos \left(\frac{2+\tilde{\theta}_{i-1}^{2}+\left(1-2 \zeta^{2}\right)^{-2}}{2 \tilde{\theta}_{i-1}}\right)
$$

where $\tilde{\theta}_{i-1}$ is the value of the function $\theta(\beta)$ at the step $i-1$. Then, by inserting $\tilde{\beta}_{i}$ into eq.(6), a new value $\tilde{\theta}_{i}$ is easily calculated:

$$
\tilde{\theta}_{i}=\frac{\left(2+\tilde{\beta}_{i}\right) \cos \left(\frac{\pi \tilde{\beta}_{i}}{2}\right)-\sqrt{\left(\left(2+\tilde{\beta}_{i}\right) \cos \left(\frac{\pi \tilde{\beta}_{i}}{2}\right)\right)^{2}-8 \tilde{\beta}_{i}}}{2 \tilde{\beta}_{i}}
$$

The iterative procedure is terminated when the difference $\left(\tilde{\theta}_{i}-\tilde{\theta}_{i-1}\right) \leq k$, with $k$ an arbitrary pre-assigned tolerance. If the convergence is not achieved, a new step is performed by going back into eq.(12); otherwise, $\beta=\tilde{\beta}_{i}$. The initial value of the function $\theta(\beta)$ necessary to start the iterative procedure can be assumed $\tilde{\theta}_{0}=1$, since $\theta(\beta) \rightarrow 1$ for $\beta \rightarrow 0$. as:

Once $\beta$ is determined, the parameter $\eta$ is easily obtained, by using the inverse of eq.(7),

$$
\eta=\theta(\beta)\left(\omega_{0} \sqrt{1-2 \zeta^{2}}\right)^{2-\beta}
$$

A numerical example is reported in Table 2. The parameters $\omega_{0}=30 \mathrm{rad} / \mathrm{sec}$ and $\zeta=0.1$ have been assigned for the target equivalent system. The $i$-th row of the table shows the values $\tilde{\beta}_{i}$ and $\tilde{\theta}_{i}$ at the $i$-th step, and the difference $\Delta \tilde{\theta}_{i}=\tilde{\theta}_{i}-\tilde{\theta}_{i-1}$ : The preassigned tolerance is $k=10^{-6}$. The final values obtained for $\beta$ and $\eta$ are reported in the last row of the table. A comparison between the target equivalent system and the determined fractional oscillator is shown in Fig.4. It should be noted that, while the proposed procedure is extremely efficient for small $\zeta$, it becomes unreliable for relatively large values of $\zeta$ (approximately $\zeta \geq 0.3$ ) since, for this case, the values of $\tilde{\beta}_{i}$ in the first few steps become larger than $\beta_{c}$, violating the initial assumptions of the proposed approach.

\begin{tabular}{lllll}
\hline STEP & $\tilde{\beta}_{i}$ & $\tilde{\theta}_{i}$ & $\Delta \tilde{\theta}_{i}$ & $\eta\left[\mathrm{sec}^{\beta-2}\right]$ \\
\hline 1 & 0.129494 & 1.02408 & $2.408 \times 10^{-2}$ & \\
2 & 0.127052 & 1.02310 & $9.777 \times 10^{-4}$ & \\
3 & 0.127185 & 1.02315 & $5.272 \times 10^{-5}$ & \\
4 & 0.127178 & 1.02315 & $2.815 \times 10^{-6}$ & \\
5 & 0.127179 & 1.02315 & $1.504 \times 10^{-7}$ & 586.282 \\
\hline
\end{tabular}

Table 2: Inverse design problem by numerical iterative procedure. 


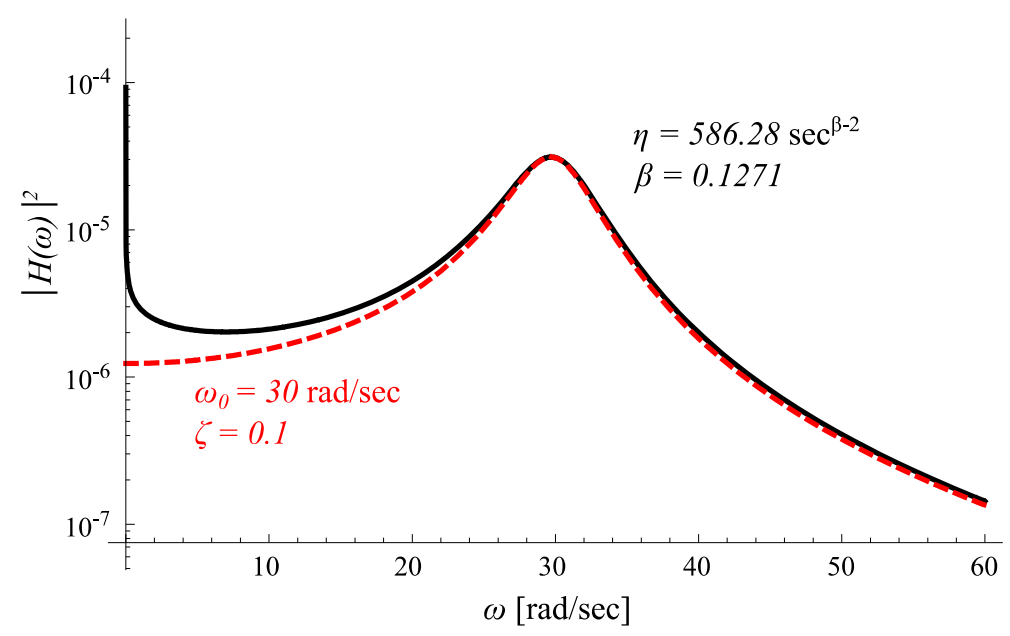

Figure 4: Transfer functions of equivalent target system (red dashed line) and fractional systems (black continuous line) reported in Table 2.

\section{VARIANCE OF THE DISPLACEMENTS OF A FRACTIONAL OSCILLATOR EXCITED BY A WHITE NOISE}

In this last section, let us consider a fractional oscillator excited by a zero-mean Gaussian white noise process. Due to the linearity of the system, a unitary one-sided Power Spectral Density function can be considered for the white noise process, without loss of generality. Then, the variance of the displacements of the fractional system is obtained as the integral in the frequency domain, between zero and infinity, of the absolute value of the transfer function $H_{f}(\omega)$ :

$$
\sigma_{f}^{2}=\int_{0}^{+\infty}\left|H_{f}(\omega)\right|^{2} d \omega
$$

Although the integral can be evaluated numerically, an analytic solution cannot be easily determined, due to the fractional nature of the transfer function $H_{f}(\omega)$. The aim of this section is to provide an approximate closed-form expression of $\sigma_{f}^{2}$ by taking advantage of the equivalent system definition proposed in the previous sections.

A first approximation of $\sigma_{f}^{2}$ can be computed in closed-form as the variance of the displacements of the equivalent system $\sigma_{e}^{2}[14]$ :

$$
\sigma_{e}^{2}=\int_{0}^{+\infty}\left|H_{e}(\omega)\right|^{2} d \omega=\frac{\pi}{4 \zeta(\beta) \omega_{0}^{3}(\eta, \beta)}
$$

where $\omega_{0}(\eta, \beta)$ and $\zeta(\beta)$ can be determined by using eqs.(10). Fig. 5 shows the comparison between the value of $\sigma_{f}^{2}$ determined by numerically computing the integral in eq.(15) and $\sigma_{e}^{2}$ evaluated by eq.(16), for $\eta=100 \sec ^{\beta-2}$ and $\beta$ varying in the range $0<\beta<\beta_{c}$. As already observed for the transfer functions of the two systems, the discrepancy between the displacement variances increases with $\beta$, while the two values coalesce when $\beta \rightarrow 0$. 


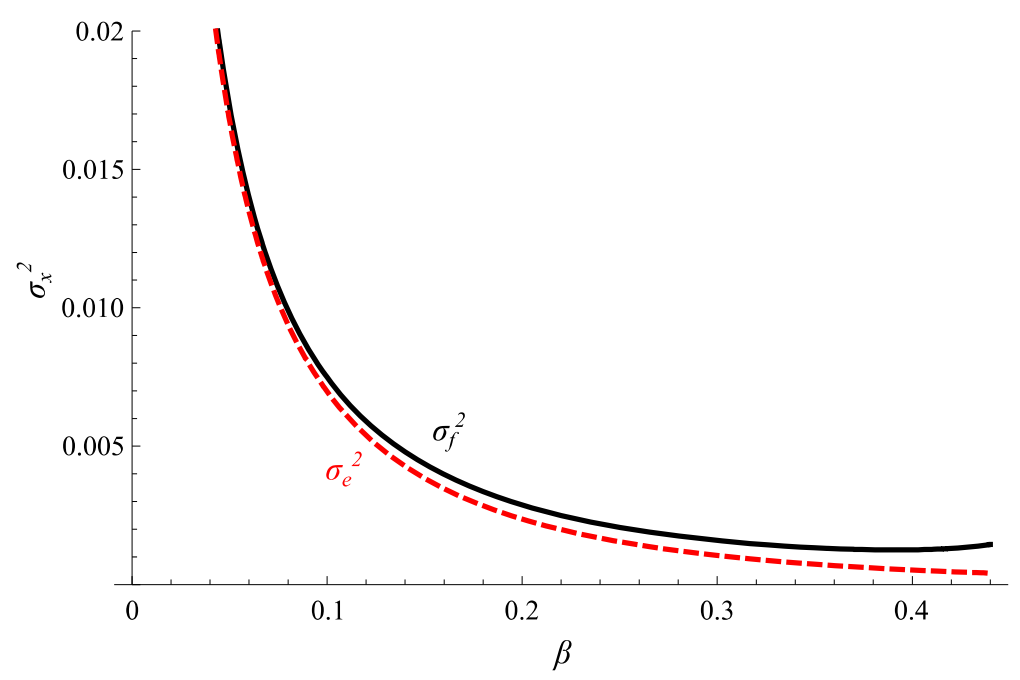

Figure 5: Variances of the displacements of fractional (continuous line) and equivalent system (dashed line).

A significant function of $\beta$ is represented by the ratio of the displacement variances of the two systems:

$$
R(\beta)=\sigma_{f}^{2} / \sigma_{e}^{2}=\frac{4 \zeta(\beta) \omega_{0}^{3}(\eta, \beta)}{\pi} \int_{0}^{+\infty}\left|H_{f}(\omega)\right|^{2} d \omega
$$

and it is shown in Fig. 6. It has been observed, by numerical simulation, that actually this function is independent of $\eta$. Although at this stage an exact expression of the function $R(\beta)$ has not been determined, the following $5^{\text {th }}$ order polynomial form is herein proposed:

$$
R(\beta)=-15.657 \beta^{5}+7.822 \beta^{4}-2.47 \beta^{3}-1.616 \beta^{2}-0.508 \beta+1
$$

The coefficients of this polynomial form have been obtained by fitting the curve shown in Fig. 6. Finally, taking advantage of eqs.(17), the variance of the fractional system can be determined in approximate closed-form as:

$$
\tilde{\sigma}_{f}^{2}=\frac{\pi R(\beta)}{4 \zeta(\beta) \omega_{0}^{3}(\eta, \beta)}
$$

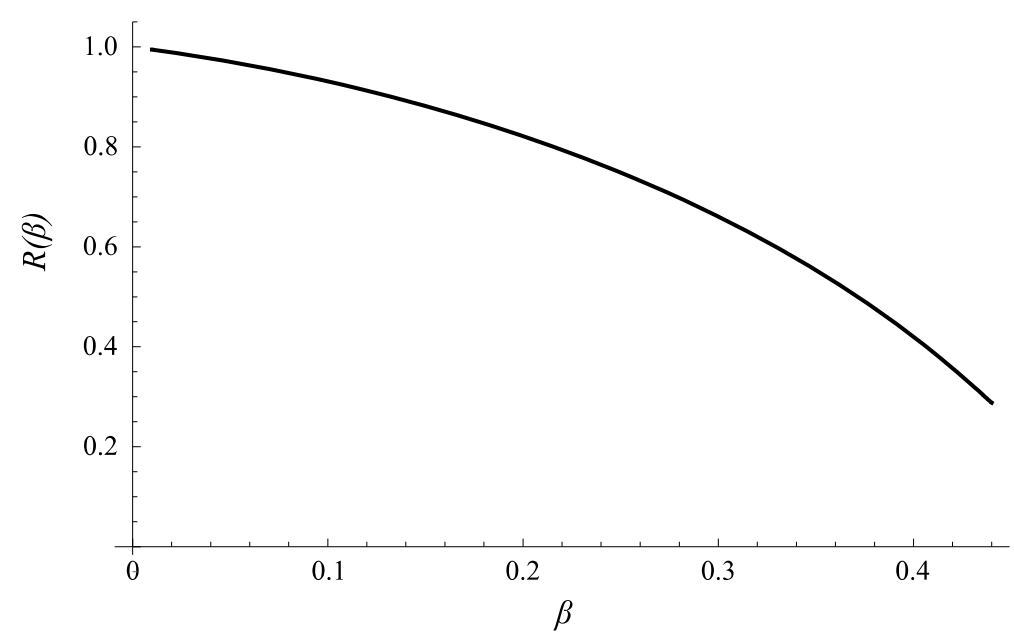

Figure 6: Ratio of the response variances of fractional and equivalent system. 
Therefore, $\sigma_{f}^{2}$ can be obtained as the variance of the displacements of a single-degree-offreedom system having natural frequency $\omega_{0}(\eta, \beta)$, to be determined by eq.(10a), and a modified damping factor $\zeta(\beta) / R(\beta)$, to be determined by eq.(10b) and eq.(18) and dependent only on $\beta$. To highlight the degree of approximation entailed by the use of eq.(19), the following error function has been defined:

$$
e(\beta)=\frac{\sigma_{f}^{2}-\tilde{\sigma}_{f}^{2}}{\sigma_{f}^{2}}
$$

where $\sigma_{f}^{2}$ is determined by numerically computing the integral in eq.(15), while $\tilde{\sigma}_{f}^{2}$ is obtained by eq.(20). The error function $e(\beta)$ is depicted in Fig. 7; it can be observed that using the proposed polynomial approximation for the function $R(\beta)$, the maximum error in the computation of $\sigma_{f}^{2}$ is about $0.15 \%$ when $\beta$ approaches the critical value $\beta_{c}$.

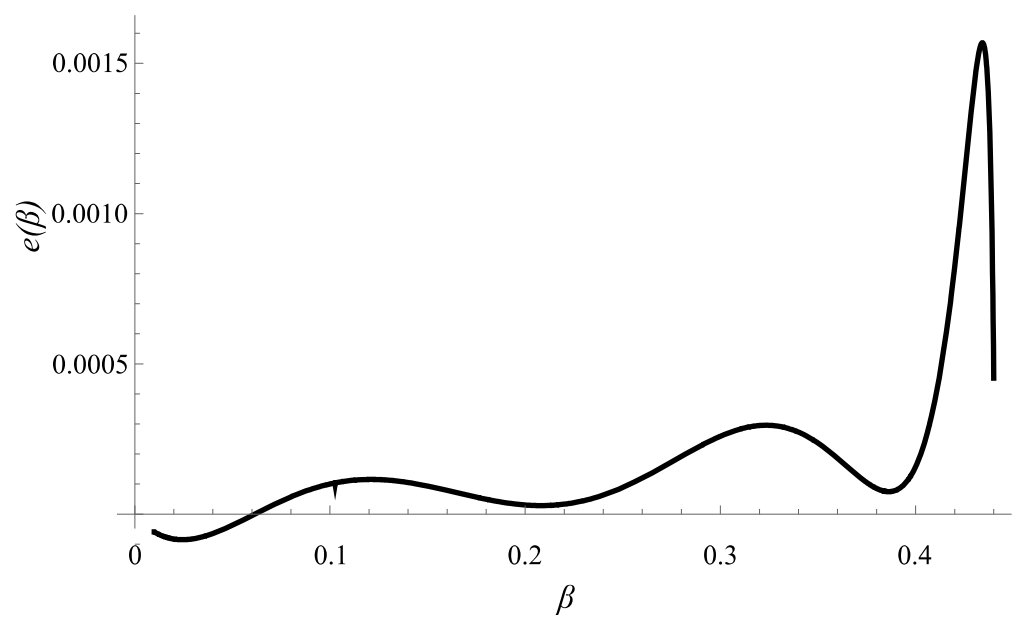

Figure 7: Error committed using eq.(19) with respect to the variance obtained by numerical integration.

\section{CONCLUSIONS}

A novel approach to the analysis of single degree-of-freedom fractional systems with dominant elastic dynamic behavior has been proposed, by defining an equivalent system with linear viscous damping. The latter is determined under the condition that the transfer functions of the two systems (fractional and equivalent) exhibit their peaks at the same frequency and with the same magnitude.

First, the direct design problem has been considered, i.e. to determine the equivalent system natural frequency and damping coefficients for an assigned fractional system. Closedform relations between the parameters of the two systems have been rigorously derived, showing that only the equivalent natural frequency $\omega_{0}$ depends on both fractional parameters, while the equivalent damping coefficient depends only on the fractional derivative order $\beta$.

Then, the inverse design problem has been analyzed, i.e. to determine the fractional system parameters for a given equivalent system. This is equivalent to design a fractional oscillator whose transfer function has a maximum for an assigned frequency and with assigned amplitude. Although for this case an analytical solution has not been provided, a numerical iterative procedure to determine the fractional parameter has been proposed.

Finally, the proposed mathematical tools have been used to determine an approximate closed-form for the variance of the displacements of the fractional oscillator excited by a zero-mean stationary Gaussian white noise. It has been shown that the maximum error committed with the proposed formulation is $0.15 \%$ with respect to the value obtained by direct numerical integration of the transfer function of the fractional system.

This paper constitutes a preliminary study of fractional systems under a new perspective; the authors are actively working to extend the proposed procedure to multi-degree-of-freedom systems and to overcome the limits imposed by the approximations related to the numerical 
approaches. The use of more sophisticated state-space viscoelastic models, e.g. the generalized Maxwell model and the Laguerre Polynomial Approximation [15] for the equivalent linear system will also be investigated.

\section{REFERENCES}

[1] P.G. Nutting, A new general law of deformation, Journal of the Franklin Institute, 191, 679-685, 1921.

[2] R.L. Bagley, P.J. Torvik, A theoretical basis for the application of fractional calculus to viscoelasticity, Journal of Rheology, 27, 201-10, 1983.

[3] N. Makris, M.C. Constantinou, Fractional derivative Maxwell model for viscous dampers, Journal of Structural Engineering, 117(9), 2708-24, 1994.

[4] R. Lewandowski, B. Chorazyczewski, Identification of the parameters of the KelvinVoigt and the Maxwell fractional models used to modeling of viscoelastic dampers, Computers and Structures, 88, 1-17, 2010.

[5] M. Di Paola, M. Zingales, Exact mechanical models of fractional hereditary materials, Journal of Rheology, 55, 982-1004, 2012.

[6] C.G. Koh, L.M. Kelly, Application of fractional derivatives to seismic analysis of base isolated models, Earthquake Engineering and Structural Dynamics, 19(2), 229-41, 1990.

[7] I. Poludbny, Fractional differential equations, Academic Press, New York, 1999.

[8] P.D. Spanos, G.I. Evangelatos, Response of a non-linear system with restoring forces governed by fractional derivatives - Time domain simulation an statistical linearization solution, Soil Dynamics and Earthquake Engineering, 30, 811-21, 2010.

[9] M. Di Paola, G. Failla, A. Pirrotta, Stationary and non-stationary stochastic response of linear fractional viscoelastic systems, Probabilistic Engineering Mechanics, 28, 85-90, 2012.

[10] G. Barone, M. Di Paola, F. Lo Iacono, G. Navarra, Viscoelastic bearings with fractional constitutive law for fractional tuned mass dampers, Journal of Sound and Vibration, 344, 18-27, 2015.

[11] G. Barone, F. Lo Iacono, G. Navarra, Dynamic characterization of fractional oscillators for Fractional Tuned Mass Dampers tuning, International Conference on "Fractional Differentiation and Its Applications” (ICFDA 2014), Catania, Italy, June 23-25, 2014.

[12] G. Barone, F. Lo Iacono, G. Navarra, Passive control of viscoelastic structures by Fractional Tuned Mass Dampers, Computational Stochastic Mechanics Conference (CSM $\left.7^{\text {th }}\right)$, Santorini, Greece, June 15-18, 2014.

[13] D.J. Inman, Engineering Vibrations, 2nd Edition. Prentice-Hall International, Inc., 2001.

[14] S.H. Crandall, W.D. Mark, Random Vibration in Mechanical Systems, Academic Press, New York, 1963.

[15] A. Palmeri, F. Ricciardelli, A. De Luca, G. Muscolino, State space formulation for linear viscoelastic systems with memory, Journal of Engineering Mechanics, 129(7), 71524, 2003. 